\title{
Cytogenetic abnormalities in 200 male infertile cases in the southern region of India*
}

\author{
G. Sreenivasa ${ }^{1}$, Suttur S. Malini ${ }^{1}$, Prasanna Kumari ${ }^{2}$, Usha R. Dutta ${ }^{3 \#}$ \\ ${ }^{1}$ Molecular Reproductive and Human Genetics Laboratory, Department of Studies in Zoology, University of Mysore, Mysore, India \\ ${ }^{2}$ Department of Pathology (Cytogenetics), Kidwai Memorial Institute of Oncology, Bangalore, India \\ ${ }^{3}$ Diagnostics Division, Center for DNA Fingerprinting and Diagnostics (CDFD), Hyderabad, India

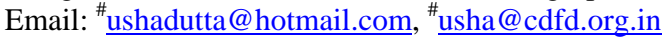

Received 21 June 2013; revised 2 July 2013; accepted 15 July 2013

Copyright (c) 2013 G. Sreenivasa et al. This is an open access article distributed under the Creative Commons Attribution License, which permits unrestricted use, distribution, and reproduction in any medium, provided the original work is properly cited.

\begin{abstract}
Chromosomal abnormalities are one of the major causes of male infertility. But the exact mechanism by which chromosomal anomalies induces infertility is still not clear. Many studies have documented the chromosomal abnormalities ranging from $2.2 \%$ to $15.7 \%$ in infertile men. The present study has been carried out to document and find out the genetic cause of male infertility in the Southern region of India. The cytogenetic analysis of 200 male infertile cases, referred due to primary infertility from 2009 to 2012, were analyzed by GTG banding and evaluated retrospectively. The semen analysis was also performed. A total of 15 cases (7.5\%) showed chromosomal aberrations. Four (2\%) were 47, XXY and mosaic 47,XXY; Two (1\%) were structural autosomal abnormalities; Two $(1 \%)$ were inversion $Y$; Seven (3.5\%) cases were $Y$ heterochromatin variants and 185 cases $(92.5 \%)$ showed normal 46,XY karyotype. The chromosomal abnormalities in our study is also in agreement with the data from the literature. Also abnormal spermatogenesis is observed in these cases. Apart from chromosomal analysis further in depth molecular analysis and genetic counseling is suggestive in such cases, especially those interested in IVF technologies.
\end{abstract}

Keywords: Male Infertility; Chromosomal Abnormalities; Semen Analysis

\section{INTRODUCTION}

Male infertility is characterized by the inability of a sex-

*Authors' Disclosures of Potential Conflicts of Interest: No potential conflicts of interest relevant to this article were reported.

\#Corresponding author. ually active, non-contraceptive couple to achieve pregnancy within one year [1]. It is a worldwide problem affecting people of all communities, though the cause and magnitude may vary with geographical location. The exact cause of male infertility is still unknown in more than $50 \%$ of cases [2]. Due to the advancement in the diagnosis technologies in genetics, it is now becoming evident that a significant percentage of male infertility cases are due to genetic abnormalities [3]. Several studies have shown increased chromosomal aberrations $[4,5]$ in $5 \%$ to $7 \%$ of patients with oligospermia, and $10 \%$ to $15 \%$ in patients with Azoospermia. Among several etiological factors, chromosomal abnormalities play a significant role in male infertility with $10 \%$ to $15 \%$ of aberrations $[6,7]$ among which $5 \%$ of these are numerical or structural abnormalities, $80 \%$ to $85 \%$ of cases are due to sex chromosome anomalies and about $2 \%$ are mosaics with autosomal abnormalities [8-11]. This value increases to about $15 \%$ in Azoospermia males, largely due to cases with 47,XXY aneuploidy. The most common type of abnormality is Klinefelter Syndrome and also Y chromosome long arm micro deletion which is described as the most frequent non chromosomal alteration [12]. Recent studies demonstrated the association between rare genetic sperm defects with a diverse inheritance pattern in the family which may be transmissible to the male offspring [13]. In our study we aimed to investigate the percentage of chromosomal abnormalities associated among infertile males in the southern region of India.

\section{MATERIALS AND METHODS}

The present study was conducted retrospectively from 2009 to 2012 in the Department of studies in Zoology of our University. Blood samples of 200 infertile subjects were collected from different IVF clinics and hospitals referred due to primary and secondary infertility. Ethical 
clearance was obtained for this study from ethical committee of University of Mysore and concerned hospital. Informed written consent was taken from all the subjects. Genetic registry was established from the subjects, which includes family history, reproductive history and life style factors. All the patients underwent a detailed physical examination and semen analysis was performed according to WHO guidelines [14]. Based on sperm count the cases were classified into different groups. Azoospermia is defined as the total absence of sperm cells and oligozoospermia is the condition which occurs when the sperm cell count is less than $5 \times 10^{6}$ cells $/ \mathrm{ml}$.

The Azoospermic group involved 48 cases, oligozoospermic group involved 19 cases, associated oligozoospermic groups like oligoasthenoteratozoosperic 17, oligoteratozoospermic 9, and oligoasthenozoospermic involved 28 cases respectively, 40 cases were recorded as teratozoospermia, 13 cases with asthenospermia, 9 cases with asthenoteratozoospermia, 10 cases with ejaculation failure and 7 cases with idiopathic condition.

Chromosomal analysis was performed on phytohemagglutinin (PHA) stimulated peripheral blood lymphocyte cultures using standard techniques [15]. GTG-banding (G-banding using Trypsin and Giemsa) was performed at approximately 400 - 450 band resolution [16]. About 50 metaphases were analyzed from each patient and up to 100 metaphases in case of mosaicism. The chromosomes were classified according to the International System for Human Cytogenetic Nomenclature (ISCN) [17].

\section{RESULT}

A total of 200 cases with male infertility were evaluated retrospectively. Fifteen out of the 200 (7.5\%) cases showed chromosomal aberrations. Four cases showed numerical sex chromosomal abnormalities (2\%). Out of which two cases showed 47,XXY, third case showed mosaic 47, XXY/46, XY and fourth case showed a mosaic karyotype of $47, \mathrm{XYY} / 46 \mathrm{XY}$. Two cases showed structural autosomal abnormalities (1\%), the first case revealed a karyotype of $46, \mathrm{XY}, \mathrm{t}(6 ; 17)(\mathrm{p} 23 ; \mathrm{p} 13)$ and the second case showed a deletion of 46,XY,del(9)(p11); Two cases showed an inversion chromosome Y (1\%), one with a karyotype of $46, X, \operatorname{inv}(\mathrm{Y})(\mathrm{p} 11.2 \mathrm{q} 11.23)$ and other showed 46,X,inv(Y)(p11.32q12). Seven cases showed Y heterochromatin variations (3.5\%) (Table 1).

Among azoospermic cases, 48 had normal karyotype (24\%) and 8 had abnormal karyotype (4\%). In this study Azoospermia condition scored 24\%. Out of the 152 Oligozoospermic cases 7 had abnormal karyotypes (3.5\%). Based on the sperm count we had subdivided them into Teratozoospermia which scored 20\%, Oligoasthenospermia which scored $14 \%$, other conditions like, Asthenospermia, Asthenoteratozoospermia, Oligoasthenoteratozoo- spermia, Idiopathic, Oligoteratozoospermia, and Ejaculation failure (Aspermia) scores less than $10 \%$. Apparently, in this study $14.5 \%$ of the Azoospermic cases, $15.7 \%$ of Oligospermic, $11.75 \%$ of Asthenoteratozoospermia, 7.8\% of Asthenospermic, 5.8\% of OAT, 5\% of Teratozoospermic and 3.5\% of Oligoasthenospermic males were found to be associated with chromosomal abnormalities (Figure 1).

The comparison of sperm parameters and the types of chromosomal abnormality among infertile males varied with the condition of the infertile subgroup. A total of 4 different numerical sex chromosomal abnormalities were observed, among them 2 cases are Klinefelter Syndrome, one case with mosaic Klinefelter and one showed the mosaic pattern of 46,XY/47,XYY were observed in the Azoospermic condition. Two cases with structural abnormalities and a total of 9 cases showing addition, deletion and inversion of $\mathrm{Y}$ chromosome in oligozoospermic condition (Table 2).

\section{DISCUSSION}

Cytogenetic abnormalities have known to cause male infertility, but the exact mechanism by which chromosomal anomalies induces infertility is still not clear. Chromosomal abnormalities include aberrations in the sex chromosomes and autosomes like insertions or deletion of an entire chromosome causing structural anomalies [18]. The sex chromosomes $\mathrm{X}$ and $\mathrm{Y}$ emerged from an autosome pair around 300 million years ago among the first mammals [19]. The Y-chromosome has become specialized in the male sex determination, progressively decreasing its gene content by losing and gaining sequences through the action of events, such as, deletions, mutations, insertions, recombination and transposition [20]. Many studies have documented the chromosomal

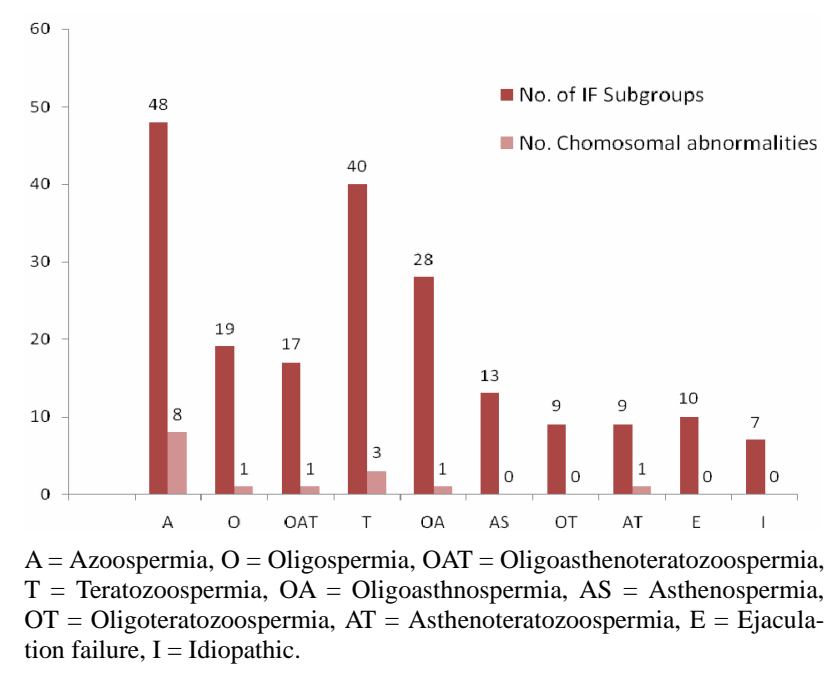

Figure 1. Distribution of infertile subgroups analyzed for chromosomal abnormalities. 
Table 1. Distribution of different types of chromosomal abnormalities among infertile males.

\begin{tabular}{|c|c|c|}
\hline Chromosomal abnormalities & $\mathbf{n}=\mathbf{2 0 0}$ & $\%$ \\
\hline Numerical sex chromosomal abnormality & 4 & 2 \\
\hline $47, \mathrm{XXY}$ & 2 & 1 \\
\hline $47, \operatorname{mos} X X Y / 46, X Y$ & 1 & 0.5 \\
\hline 47,mosXYY/46,XY & 1 & 0.5 \\
\hline Structural autosomal chromosomal abnormality & 2 & 1 \\
\hline 46,XY,t(6;17)(p23;p13) & 1 & 0.5 \\
\hline 46,XY,del(9)(p11) & 1 & 0.5 \\
\hline Structural Y chromosome abnormality & 09 & 4.5 \\
\hline 46,XYq12h & 4 & 2.0 \\
\hline 46,XYq12h- & 3 & 1.5 \\
\hline 46,X,inv(Y)(p11.2q11.23) & 1 & 0.5 \\
\hline $46, X, \operatorname{inv}(Y)(p 11.32 q 12)$ & 1 & 0.5 \\
\hline Normal 46,XY & 185 & 92.5 \\
\hline
\end{tabular}

Table 2. Cytogenetic abnormalities with respect to the sperm parameters in different infertile subgroups, IF = Infertile.

\begin{tabular}{|c|c|c|c|c|}
\hline Karyotype $(n=15)$ & IF & Sperm count (millions/ml) & Motility & Morphology \\
\hline $47, X X Y$ & Azo & 0 & $0 \%$ & $0 \%$ \\
\hline 47,XXY & Azo & 0 & $0 \%$ & $0 \%$ \\
\hline $\operatorname{mos} 47, X Y Y / 46, X Y$ & Azo & 0 & $0 \%$ & $0 \%$ \\
\hline $\operatorname{mos} 47, \mathrm{XXY} / 46, \mathrm{XY}$ & Azo & 0 & $0 \%$ & $0 \%$ \\
\hline $46, X Y q 12 h-$ & Azo & 0 & $0 \%$ & $0 \%$ \\
\hline $46, \mathrm{XYq} 12 \mathrm{~h}-$ & OAT & 10 & $5 \%$ & $40 \%$ \\
\hline 46,XYq12h- & Azo & 2 & $15 \%$ & $20 \%$ \\
\hline $46, X Y q 12 h$ & $\mathrm{OA}$ & 5 & $0 \%$ & $40 \%$ \\
\hline 46,XYq12h & Azo & 0 & $0 \%$ & $0 \%$ \\
\hline 46,XYq12h & $\mathrm{T}$ & 0 & $0 \%$ & $0 \%$ \\
\hline 46,XYq12h & $\mathrm{T}$ & 98 & $65 \%$ & $15 \%$ \\
\hline $46, X, \operatorname{inv}(Y)(p 11.2 q 11.23)$ & Azo & 0 & $0 \%$ & $0 \%$ \\
\hline $46, X, \operatorname{inv}(Y)(p 11.32 q 12)$ & $\mathrm{T}$ & 85 & $60 \%$ & 15 \\
\hline 46,XY,t(6;17)(p23;p13) & AT & 58 & $0 \%$ & 10 \\
\hline 46,XY,del(9)(p12) & $\mathrm{O}$ & 5 & $55 \%$ & 21 \\
\hline
\end{tabular}

abnormalities which ranges from $2.2 \%$ to $15.7 \%$ for infertile men [18] in our study we observed that $7.5 \%$ of the infertile males are associated with chromosomal abnormality.

Sex chromosome aberrations are the most frequently observed conditions in male infertility. Klinefelter syndrome males can be identified only when they undergo fertility assessments. VanAssche et al. [21] reported 11\% Azoospermic individuals with Klinefelter syndrome. In our study among 48 (24\%) of the Azoospermia cases, $4 \%$ cases were true Klinefelter syndrome, $2 \%$ with mosaic Klinefelter syndrome. The occurrence of Klinefelter syndrome and XYY is due to meiotic non disjunction of the $\mathrm{X}$ chromosome or anaphase lag of $\mathrm{X}$ chromosome from a normal 46,XY or XXY zygote. This abnormality is associated with severe spermatogenic failure causing a marked reduction in testicular size and resulting in Azoospermia [22]. Earlier studies have reported that the men with XYY syndrome are generally fertile but appear to have an increased likelihood of infertility compared to karyotypically normal 46,XY males. This type of chromosomal anomaly occurs in one for 1000 live male births in the general population, but more frequent in the infertile population (Martin, 2008). We also had one case with a frequency of $0.5 \%$ associated with Azoospermic condition having mosaic 46,XY/47,XYY karyotype. Few 
studies also reported the association of infertile patients with 47,XYY syndrome [23]. Among Klinefelter and $47, \mathrm{XYY}$ syndrome males, there is a theoretical risk regarding the manifestation of sex chromosomal aneuploidy in at least $50 \%$ of their sperms [24].

Recent study reported the higher incidence of chromosomal abnormalities in azoospermic group than in the oligospermic groups and also increased chromosomal abnormalities with respect to decreased sperm count. Some researchers who had investigated chromosomal anomalies, specifically among patients with severe oligospermia and Azoospermia, have reported higher figures such as $20.86 \%$ [11] and $21.1 \%$ [25] reported that the incidence of chromosomal abnormalities was $14.3 \%$ and 6.5\% among Azoospermia and oligospermia respectively, also Ceylan et al. [26] reported that, chromosomal abnormality was $33.3 \%$ in the azoospermic and $13.3 \%$ in severe oligozoospermic group. Chromosomal abnormalities were detected in $17.4 \%$ of 86 azoospermic cases and in $6.8 \%$ of 73 oligozoospermic cases in a regional study in Turkey [27]. According to Samli et al. [28] chromosomal abnormality were associated with $12 \%$ of azoospermic cases and in $4 \%$ of oligospermic patients. In our study the incidence of the chromosomal abnormalities in azoospermic and oligospermic males were found to be $14.5 \%$ and $5.2 \%$ respectively which accords with previous studies.

Additionally, the present study showed $2 \%$ of the associated oligospermic cases, like Oligo Asthenospermia and oligoasthenoteratozoospermia condition showed Y chromosomal heterochromatin variation with a frequency of $1.2 \%$ have associated severe sperm defects. A relationship between balanced autosomal translocation and infertility has been reported. In the present study one case with $(0.5 \%) 46, \mathrm{XY}, \mathrm{t}(6 ; 17)(\mathrm{p} 23 ; \mathrm{p} 13)$ and one case with deletion of 9p12 was observed. Many studies reported that, the association of chromosome 9 in inversion, translocation, and other chromosomal aberrations with the male infertility which may result in various clinical manifestations and also cause different kinds of sperm abnormalities $[27,29]$. In the majority of cases, carriers of balanced translocations are themselves phenotypically normal, unless one of the translocation breakpoints interrupts an important gene or via position effect.

\section{CONCLUSION}

Chromosomal abnormalities play a significant role in male infertility. In the present study it has been found that the infertile males associated with chromosomal abnormalities involving structural as well as numerical alterations might have affected the spermatogenesis. However, further indepth Molecular analysis is needed to understand the additional genetical factors of male infertility.

\section{ACKNOWLEDGEMENTS}

GS would like to thank UGC-RFSMS for the financial assistance.

\section{REFERENCES}

[1] WHO (2000) WHO manual for the standardized investigation and diagnosis of the infertile couple. Cambridge University Press, Cambridge.

[2] Dada, R. and Gupta, N.C. (2004) Yq microdeletions-Azoospermia factor candidate genes and spermatogenetic arrest. Journal of Biomolecular Techniques, 15, 176-183.

[3] Maduro, M.R. and Lamb, D.J. (2002) Understanding new genetics of male infertility. Journal of Urology, 68, 21972205.

[4] Retief, A.E., Van Zyl, J.A., Menkveld, R., Fox, M.R., Kotze, G.M. and Brusnicky, J. (1984) Chromosome studies in 496 infertile males with a sperm count below 10 million/ml. Human Genetics, 66, 162-164. doi:10.1007/BF00286592

[5] Ravel, C., Berthaut, I., Bresson, J.L. and Siffroi, J.P. (2006) Prevalence of chromosomal abnormalities in phenotypically normal and fertile adult males: Large-scale survey of over 10000 sperm donor karyotypes. Human Reproduction, 21, 1484-1489.

doi:10.1093/humrep/del024

[6] Penna, V.S., Araujo, H., Ballesta, F., Ballesca, J.L. and Vanrell, J.A. (2001) Chromosomal abnormalities and polymorphisms in infertile men. Archives of Andrology, 46, 205-210. doi:10.1080/01485010151096504

[7] Patsalis, P.C., Sismani, C., Quintana-Murci, L., Taleb-Bekkouche, F., Krausz, C. and McElreavey, K. (2002) Effects of transmission of $\mathrm{Y}$ chromosome AZFc deletions. The Lancet, 360, 1222-1224. doi:10.1016/S0140-6736(02)11248-7

[8] Siffroi, J.P., Le Bourhis, C., Krausz, C., Barbaux, S., Quintana-Murci, L. and Kanafani, S. (2005) Sex chromosome mosacism in males carrying Y chromosome long arm deletions. Human Reproduction, 15, 2559-2562. doi:10.1093/humrep/15.12.2559

[9] Visootsak, J., Aylstock, M. and Graham, J.M. (2001) Klinefelter syndrome and its variants: An update and review for the primary pediatrician. Clinical Pediatrics, 40, 639-651. doi:10.1177/000992280104001201

[10] Huynh, T., Mollard, R. and Trounson, A. (2002) Selected genetic factors associated with male infertility. Human Reproduction, 8, 183-198. doi:10.1093/humupd/8.2.183

[11] Zhou, H., Zhu, J.W., Li, H.G. and Tang, Y.P. (2009) Genetic defect in Chinese azoospermic patients and their relationship with reproductive hormones. Chinese Journal of Medical Genetics, 26, 427-430.

[12] Yoshida, A., Miura, K. and Shirai, M. (1996) Chromosome abnormalities and male infertility. Assisted Reproduction Reviews, 6, 93-100.

[13] Baccetti, B., Capitani, S., Collodel, G., Di Cairano, G., Gambera, L., Moretti, E. and Piomboni. P. (2001) Genetic sperm defects and consanguinity. Human Reproduction, 16, 1365-1371. 
[14] World Health Organization (1999) Laboratory manual for the examination of human semen and sperm-cervical mucus interaction. Cambridge University Press, New York.

[15] Moorhead, P.S., Nowell, P.C. and Mellman, W.J. (1960) Chromosome preparations of leukocytes cultured from human peripheral blood. Experimental Cell Research, 20, 613-616. doi:10.1016/0014-4827(60)90138-5

[16] Seabright, M. (1971) A rapid banding technique for human chromosomes. Lancet, 2, 971-972. doi:10.1016/S0140-6736(71)90287-X

[17] ISCN (2009) An international system for human cytogenetic nomenclature.

[18] Azimi, C., Khaleghian, M. and Farzanfar, F. (2012) Cytogenetic studies among Iranian infertile men: The first 20-year long-term report. African Journal of Biotechnology, 11, 8973-8978.

[19] Lahn, B.T., Pearson, N.M. and Jegalian, K. (2001) The human Y chromosome, in the light of evolution. Nature Reviews Genetics, 2, 207-216. doi:10.1038/35056058

[20] Charlesworth, D., Charlesworth, B. and Marais, G. (2005) Steps in the evolution of heteromorphic sex chromosomes. Heredity, 95, 118-128. doi:10.1038/sj.hdy.6800697

[21] VanAssche, E., Bonduelle. M., Tournaye, H., Joris, H., Verheyen, G., Devroey, P, Van Steirteghem, A. and Liebaers, I. (1996) Cytogenetics of infertile men. Human Reproduction, 11, 1-26. doi:10.1093/humrep/11.suppl_4.1

[22] Pandiyan, N. and Jequier, A.M. (1996) Mitotic chromosomal anomalies among 1210 infertile men. Human Reproduction, 11, 2604-2608. doi:10.1093/oxfordjournals.humrep.a019178
[23] El-Dahtory, F. and Elsheikha, H.M. (2009) Male infertility related to an aberrant karyotype, 47, XYY: Four case reports. Cases Journal, 2, 28. doi:10.1186/1757-1626-2-28

[24] Yoshida, A., Nakahori, Y., Kuroki, Y., Motoyama, M., Araki, Y., Miura, K., et al. (1997) Dicentric Y chromosome in an azoospermic male. Molecular Human Reproduction, 3, 709-712. doi:10.1093/molehr/3.8.709

[25] Paulina, P.Y., Mary, H.Y., Elizabeth, T., Lucy, K.L., Ernest, H.Y., William, S.B. and Yeung, P.C. (2009) Chromosomal anomalies and Y-microdeletions among Chinese subfertile men in Hong Kong. Hong Kong Medical Journal, 15, 31-38.

[26] Ceylan, G.G., Ceylan, C. and Elyas, H. (2009) Genetic anomalies in patients with severe oligozoospermia and azoospermia in eastern Turkey: A prospective study. Genetics and Molecular Research, 8, 915-922. doi:10.4238/vol8-3gmr616

[27] Akgul, M., Ozkinay, F., Ercal, D., Cogulu, O., Dogan, O., Altay, B., Tavmergen, E., Gunduz, C. and Ozkinay, C. (2009) Cytogenetic abnormalities in 179 cases with male infertility in Western Region of Turkey: Report and review. Journal of Assisted Reproduction and Genetics, 26, 119-122. doi:10.1007/s10815-009-9296-8

[28] Samli, H., Samli, M.M., Solak, M. and Imirzalioglu, N. (2006) Genetic anomalies detected in patients with nonobstructive azoospermia and oligozoospermia. Archives of Andrology, 52, 263-267. doi:10.1080/01485010600664032

[29] Nagvenkar, P., Desai, K., Hinduja, I. and Zaveri, K. (2005) Chromosomal studies in infertile men with oligozoospermia \& non-obstructive azoospermia. Indian Journal of Medical Research, 122, 34-42. 